\title{
Sequence analysis and evaluation of the NS3/A gene region of BTV isolates from South
}

\section{Africa}

Steyn J. and Venter E. H.

Corresponding author:

Miss Jumari Steyn

Tel: +27 123192352

Cell: +27826916555

jumari.steyn@gmail.com

Department of Veterinary Tropical Diseases, Faculty of Veterinary Science, University of Pretoria, Onderstepoort, South Africa

Author contribution: Generating of results, analysis and write up.

Prof Estelle. Hildegard Venter

estelle.venter@up.ac.za

Tel:

Cell: +27836337284

Private Bag X04, Onderstepoort 0110, South Africa

Department of Veterinary Tropical Diseases, Faculty of Veterinary Science, University of Pretoria, Onderstepoort, South Africa

Author contribution: Supervisor; funding and editing.

\section{Compliance with Ethical Standards}

This study was approved by the Research Committee (V079/12)

Note: Nucleotide sequence data reported are available in the GenBank databases under the accession numbers: BTV-2 is KJ506696

BTV-7 is KJ506695

BTV-8 is KJ506703

BTV-11 is KJ506694

BTV-16 is KJ506697

BTV-19 is KJ506699

BTV-20 is KJ506702

BTV-21 is KJ506701

BTV-22 is KJ506698

BTV-22 is KJ506700

BTV-S299 is KJ506704 


\begin{abstract}
Phylogenetic networks and sequence analyses allow for a more accurate understanding of the serotype, genetic relationships and the epidemiology of viruses. Based on gene sequences of the conserved segment 10 (NS3), bluetongue virus can be divided into five topotypes. In this molecular epidemiology study, segment 10 sequence data of 11 isolates obtained from the Virology Section of the Department of Veterinary Tropical Diseases, Faculty of Veterinary Science, University of Pretoria, Onderstepoort, were analyzed and compared to sequence data of worldwide BTV strains available on GenBank. The consensus nucleotide sequences of NS3/A showed intermediate levels of nucleotide variation with a nucleotide identity ranging from $79.72 \%$ to $100 \%$. All 11 strains demonstrated conserved amino acid characteristics. Phylogenetic networks were used to identify BTV topotypes. The phylogeny obtained from the nucleotide sequence data of the NS3/A-encoding gene presented three major and two minor topotypes. The clustering of strains from different geographical areas into the same group indicated spatial spread of the segment 10 genes, either through gene reassortment or through the introduction of new strains from other geographical areas via trade. The effect of reassortment and genetic drift on BTV and the importance of correct serotyping to identify viral strains are highlighted.
\end{abstract}

\title{
Introduction
}

During the last decade bluetongue virus (BTV) has become a major concern worldwide as well as the focus point of many epidemiological studies and surveillance programs. The virus is the prototype member of the genus Orbivirus in the family Reoviridae and causes an economically important infectious, non-contagious disease in domestic and wild ruminants called bluetongue (BT) [1,2]. Twenty six serotypes [3-6] of the virus have been identified, that are transmitted primarily by certain species of biting midges that belong to the genus Culicoides (Diptera: Ceratopogonidae) [4]. Recent findings related to BTV-8 circulation in Europe have shown that the occurrence of viral transmission is not only via biting midges, but also directly from ruminant to ruminant by transplacental transmission [8-10]. In addition, Menzies., et al [11] have documented the transmission of a field strain of BTV-8 via transplacental and probably the oral route.

Molecular phylogenetic analyses are imperative in epidemiological studies. Phylogenetics have been used widely to identify and categorize different viruses such as Newcastle disease virus [12], avian influenza A virus [13], foot-and-mouth disease virus (FMDV) [14] and Dengue virus [15]. Methods can be used to determine the origin of viruses, source of outbreaks and spread that could assist in the control of a disease [16-18].

Phylogenetic networks that include phylogenetic trees and split networks are important in the reconstruction of evolutionary history [19]. Split networks are gaining popularity because of the exploration and visualization of different genetic differences such as reassortment in closely related taxa that is not easily visualized with traditional methods [19]. Split networks such as the neighbour-net analysis are capable of representing incompatible and ambiguous signals in data sets by allowing additional splits with weights. This allows split networks to fit the data better than a single bifurcating tree, considering that weights are analogous to the length of a branch in a phylogenetic tree [19]. Reassortment, genetic drift and shift have been identified as key factors responsible for the evolution of various segmented genome viruses that may lead to novel viruses $[15,13,12]$. 
During a recent BTV outbreak in northern Europe, phylogenetic analyses were used to identify the origins of the responsible viruses $[20,16]$. Sequence analysis revealed that several of the viruses originated in sub-Saharan Africa, including a South African vaccine strain $[17,18]$. The release of such vaccine strains has increased the level of genetic diversity within the BTV field population and emphasized the complexity of the epidemiology of BTV [20].

The segmented nature of the BTV genome allows for reassortment within the host or vector when either is simultaneously infected with two or more strains [21, 22]. This has complicated the genetic classification of BTV to some extent and it is desirable to conduct viral topotyping through sequence analyses of more than one gene segment [23]. Arthropod-borne RNA viruses that rely on both a vertebrate host and an insect vector have demonstrated slower rates of evolution compared to single-host RNA viruses. This might be due to the different selective pressures present in two very different environments in two hosts [24-26].

The BTV non-structural protein NS3/A has been associated with the release of virus particles from insect cells $[27,28]$. It has been suggested that the variations observed in this protein may relate to transmission of the virus by distinct insect vector species in different geographical regions [29]. No evidence of co-evolution in the segment 10 gene has been demonstrated, only negative selection due to genetic drift and the founder effect [21, $30]$.

Segment 10 of the BTV genome codes both NS3 and NS3A proteins. These are intermediately conserved with a nucleotide sequence identity of more than $80 \%$ across all isolates, independent of serotype [20]. The NS3/A protein has conserved characteristics shared by several orbiviruses, e.g. BTV, African horse sickness virus (AHSV) and equine encephalosis virus (EEV) [31]. These regions include a second in-phase start codon, a proline-rich domain and hydrophobic regions [32]. Based on evolutionary distinct geographical variants and phylogenetic analyses obtained from segment 10, BTV can be divided into three Eastern and two Western topotypes [30, 20,33].

In this study sequence data from BTV isolates were compared to establish the topotypes of newly sequenced strains using phylogenetic networks. The highly conserved NS3/A-encoding gene region was compared to NS3/A sequences of strains representative of different geographical regions globally. This could give us an indication of the degree of variability between BTV strains and add to the knowledge of the epidemiology of BTV in South Africa. 


\section{Materials and Methods}

Bluetongue viral strains

A total of 11 viral strains dated from 1972 to 2000 and labelled 'reference strains' by the Department of Veterinary Tropical Disease, University of Pretoria were used in this study.

Cell culture

Bluetongue virus isolates were cultivated in African green monkey kidney (Vero) cells. Vero cells were grown in Dulbecco's minimal essential medium (MEM) (containing Earle's salts and $\left.\mathrm{NaHCO}_{3}\right)($ Highveld Biological (Pty) LTD) supplemented with 10\% gamma-irradiated foetal calf serum (v/v) (Highveld Biological (Pty) LTD), $10 \%$ tryptose phosphate broth (v/v) (Sigma-Aldrich Co. LLC), $1 \mathrm{ml}$ Gentamycin $(500 \mu \mathrm{g} / \mathrm{ml})$ (Virbac Animal Health) and $0.125 \mathrm{ml}$ Fungizone $(10 \mathrm{mg} / \mathrm{ml})$ (Bristol-Myers Squibb) in tissue culture flasks (containing plug seal caps) at $37^{\circ} \mathrm{C}$ for seven days until confluent. Confluent flasks were infected with each of the 11 BTV field isolates and incubated for 7 days or until $100 \%$ cytopathic effect were observed. Cell cultures were frozen and thawed three times after which cell debris were collected by centrifugation for 10 minutes at $2000 \mathrm{~g}$.

\section{$\underline{\text { Viral RNA extraction }}$}

Total RNA was extracted using an Express Magnetic Particle Processor, the MagMAX ${ }^{\mathrm{TM}}$ Express (Ambion $\left.{ }^{\circledR}\right)$, using the MagMAX ${ }^{\mathrm{TM}}$ Total Nucleic Acid Isolation Kit (Applied Biosystems part number AM1830). This kit is designed for rapid, high-throughput purification of RNA and combines an effective sample disruption method with magnetic bead-based nucleic acid purification technology. Extractions were performed according to the manufacturer's instructions.

\section{$\underline{\text { RT-PCR/ amplification }}$}

Extracted double stranded RNA was denatured by heating for 3 minutes at $95^{\circ} \mathrm{C}$ and placing it at $-20^{\circ} \mathrm{C}$ for 5 minutes. Reverse transcription was carried out with the GeneAmp® Gold RNA PCR Reagent Kit (Applied Biosystems®) using segment 10-specific forward (5'-GTTAAAAAGTGTCGTGCC-3') and reverse (5'GTTAGTGTGTAGAGCCGCG-3') primers according to manufacturer's instructions. Amplification of cDNA was performed using Takara ${ }^{\circledR}$ Taq in combination with the same segment 10 specific primers with the thermocycler (Applied Biosystems ${ }^{\circledR}$ ) according to manufacturer's instructions.

\section{$\underline{\text { DNA sequencing and analysis }}$}

Amplified PCR products were sent to Inqaba Biotechnical Industries (Pty) Ltd for purification and Sanger sequencing. The purified DNA was sequenced in both directions using the same primers as in the PCR amplification step. Resulting sequence data were edited and analysed using the Staden Package (http://staden.sourceforge.net/), while multiple sequence alignments of the NS3/A-encoding gene and translated amino acid sequences were done using default parameters of CLUSTAL X (version 2.1) and BioEdit (version 7.2.5). Bluetongue virus sequences were selected based on serotype and downloaded from GenBank as well as 
sequence data from another member of the Orbivirus genus, epizootic hemorrhagic disease virus (EHDV), to use as an out-group to root the trees (Appendix I). The number of nucleotide differences between BTV strains was determined using pairwise distance with 1000 bootstrap replicates in MEGA 6 (Molecular Evolutionary Genetics Analysis software). Conserved regions were identified and amino acids positions were compared between strains. The best nucleotide substitutions model was determined using MEGA 6. The model with the lowest Bayesian Information Criterion (BIC) i.e., Tamura-3 using a gamma distribution (T92+G) [34], was used to construct a neighbour-joining (NJ) tree with 1000 bootstrap replicates. A splits network inferring a neighbour-net analysis was used to extract phylogenetic signals that are missed by tree-based methods and to exclude any systematic error [35]. This analysis was undertaken in SplitsTree 4 [19].

\section{Results}

The NS3/A gene of 11 isolates were amplified and sequenced successfully (Appendix II), which included original isolates, vaccine strains and reference antigens. PCR products were viewed by agarose gel electrophoresis and sequence data were uploaded to NCBI GenBank to obtain accession numbers (Appendix II). All of the BTV segment 10 sequences analysed were $>800$ nucleotides, with the NS3/A start codon (ATG) at nucleotide position 40 to 42 . The open reading frame of the NS3/A protein varied between $648-690$ nucleotides and the NS3/A protein ranged from 215 - 229 amino acids. The differences are due to partial sequencing of some of the isolates.

\section{Nucleotide variation}

Nucleotide variation of newly sequenced strains relevant to this study (Appendix II) has been described and compared (Table I). Segment 10 or the NS3/A-encoding gene show intermediate levels of nucleotide variation between isolates with a nucleotide identity that ranged from $100 \%$ to $79.72 \%$ (Table I).

\section{$\underline{\text { Phylogenetic network analyses }}$}

There was no difference in topotyping between the two phylogenetic network methods (NJ and neighbour-net analyses), suggesting that there was no error in the tree-based phylogenetic model. The NJ tree (Fig. 1) revealed two distinct monophyletic lineages with $72 \%$ (Fig. 1a) and $86 \%$ (Fig. 1c) confidence levels, and three minor lineages [Fig. 1 (a, b and c)] with confidence levels of 68\%, 98\% and 93\% (Fig. 1b). The segregation of segment 10 corresponded to clusters determined previously i.e. Western group 1, 2 and 3 and Eastern group 1 and 2 [30, 20]. The neighbour-net tree [Fig. 2 (a - e)] also provided strong evidence for networked evolution among BTV strains.

Segment 10 of BTV-2 from Italy (JN255871), France (AF481093) and SA (AF512920), BTV-7 from USA (FJ713322) and SA (JX272558), BTV-8 from SA (AF512919, AF512924 and JX272548) and the Caribbean (AY426604), BTV-11 from SA (AF512921, AF512923, AF512922 and JX272518), Germany (JQ972860), Belgium (JQ972850 and JQ972840) and USA (AF044377 and AF044373) and -22 from SA (JX272398), along with the sequences obtained from this study, BTV-2 (KJ506696), BTV-7 (KJ506695), BTV-11 (KJ506694), BTV-22 (KJ506698) and BTV-16 (KJ506697) clustered together in Western group 2 (Fig. 1a, Fig. 2a). The 
Table I: Comparison of nucleotide sequences between newly sequenced BTV segment 10 and reference strains obtained from GenBank. The number of differences between strains is presented as a percentage. Most nucleotide differences (upper percentile) and the least number of nucleotide differences (lower percentile) are given in the table.

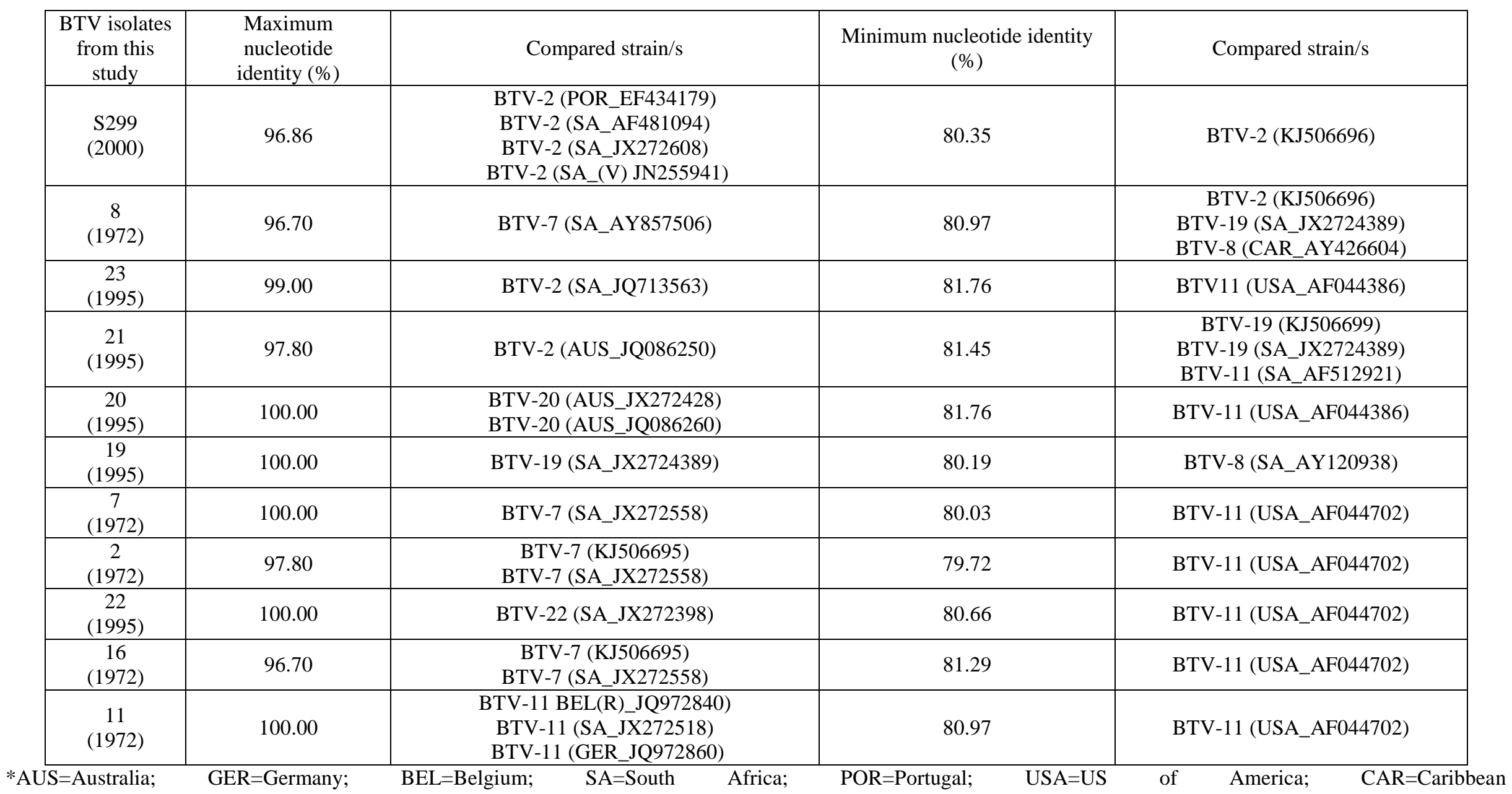




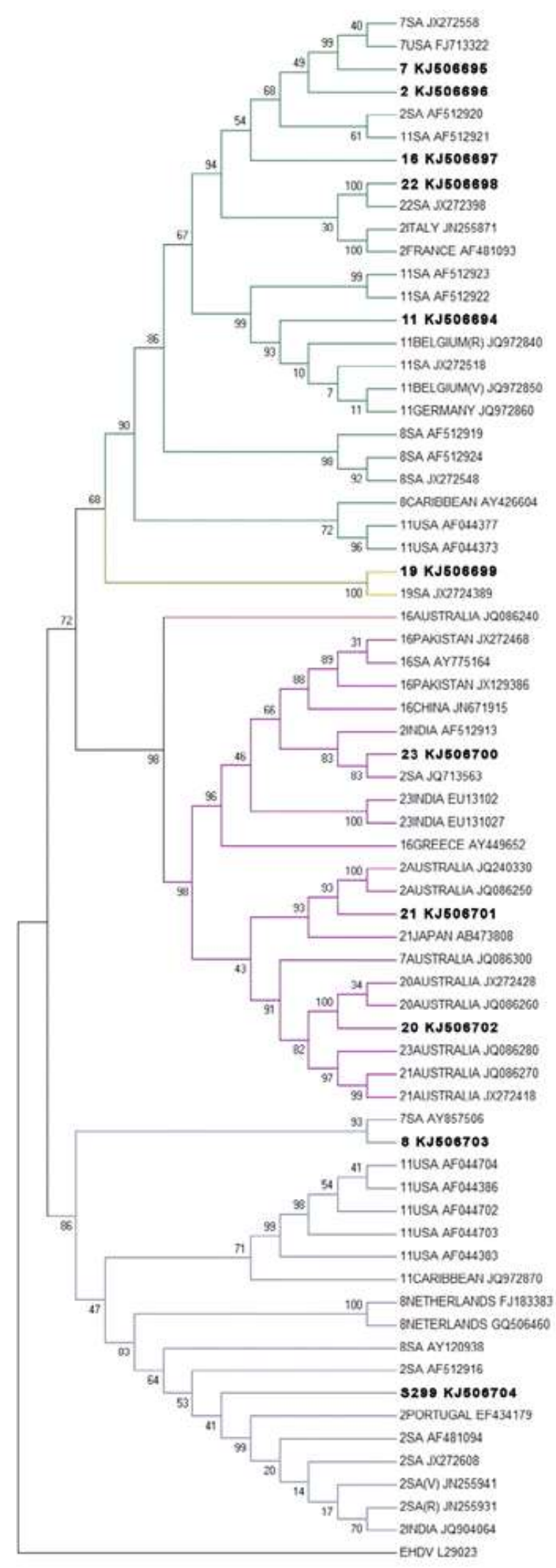

(a) Eastern group 1 \& 2:

Australia, Asia, Europe and SA (b) Eastern group 1 \& 2:

Australia, Asia, Europe and SA (c) Western group 1:

SA, USA, Caribbean and Europe

Fig. 1 : Neighbour-joining tree (calculated using BI, with the T92+G substitution model and bootstrap estimates from 1000 replicates), showing the relationships of 67 BTV segment 10 nucleotypes using EHDV as an out-group (black). The tree reveals three major groups, i.e., western 1 (c: blue) and 2 (a: green) and eastern group 1 (b: purple), and two minor groups, i.e., western 3 (c: yellow) and eastern 2 (b: red). Confidence scores are shown on the branches. Bold names represent newly sequenced BTV strains 


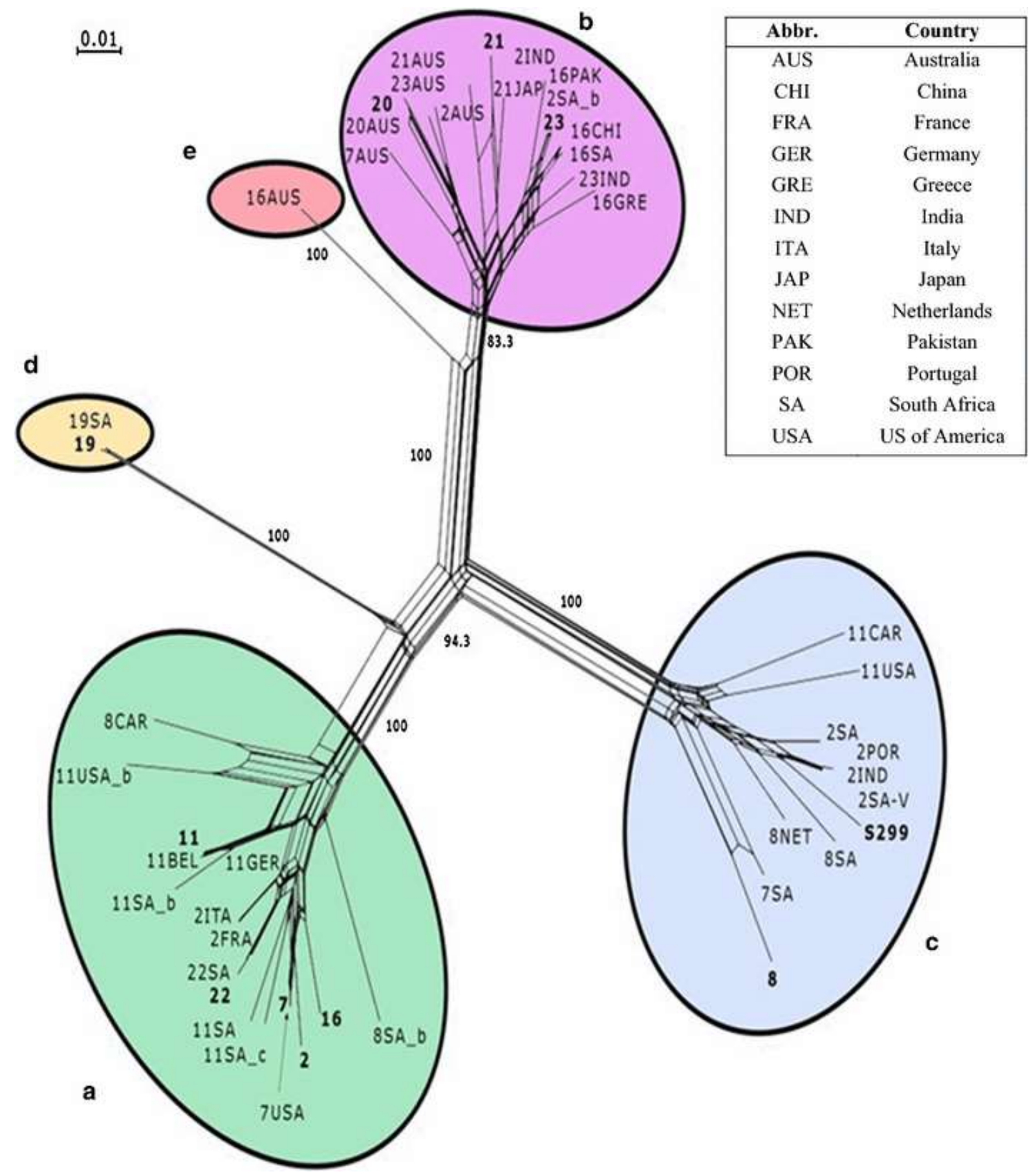

Fig. 2 : Neighbour-joining tree (calculated using BI, with the T92+G substitution model and bootstrap estimates from 1000 replicates), showing the relationships of 67 BTV segment 10 nucleotypes using EHDV as an out-group (black). The tree reveals three major groups, i.e., western 1 (c: blue) and 2 (a: green) and eastern group 1 (b: purple), and two minor groups, i.e., western 3 (c: yellow) and eastern 2 (b: red). Confidence scores are shown on the branches. Bold names represent newly sequenced BTV strains

minor lineages, Western group 3, consist of BTV-19 (JX2724389) and BTV-19 (KJ506699) from SA (Fig. 1a, Fig. 2d).

Segment 10 of BTV-2 from Australia (JQ240330 and JQ086250), India (AF512913) and SA (JQ713563), BTV7 from Australia (JQ086300), BTV-16 from China (JN671915), Pakistan (JX129386 and JX272468), SA 
(AY775164) and Greece (AY449652), BTV-20 from Australia (JX272428 and JQ086260), BTV-21 from Australia (JQ086270 and JX272418) and Japan (AB473808), BTV-23 from Australia (JQ086280) and India (EU13102 and EU131027) along with newly sequenced strains BTV-20 (KJ506702), BTV-21 (KJ506701) and BTV-23 (KJ506700) clustered together in Eastern group 1 (Fig. 1b, Fig. 2b), while segment-10 of BTV-16 from Australia (JQ086240) formed the minor Eastern group 2 lineage (Fig. 1b, Fig. 2.3e).

Segment 10 of BTV-2 from India (JQ904064), SA (AY120938, AF512916, JN255931, AF481094, JX272608 and JN255941) and Portugal (EF434179), BTV-8 from the Netherlands (FJ183383 and GQ506460), BTV-7 from SA (AY857506), and BTV-11 from USA (AF044704, AF044386, AF044703, AF044702 and AF044383) and Caribbean (JQ972870) as well as two of the sequences of interest, BTV-S299 (KJ506704) and BTV-8 (KJ506703) cluster together in Western group 1 (Fig. 1c, Fig. 2c).

\section{$\underline{\text { Amino acid sequence analyses }}$}

The BTV segment 10 sequences were conserved between the isolates sequenced in this study. However, some isolates demonstrated unique amino acids changes, but overall the isolates were $76 \%$ conserved at the amino acid level. All 11 sequences demonstrated a conserved cysteine (C) at amino acid positions 137 and 181, and a conserved tryptophan (W) residue at amino acid position 159 (Fig. 3). Potential $\mathrm{N}$-linked glycosylation sites are present at amino acid position 63 to 65 (NTT) for all isolates, while the N-linked glycosylation sites at position 150 to 152 (NGT) were conserved in 10 of the viruses, albeit that BTV-19 (KJ506699) contains a NNA sequence instead. Other regions present in all 11 isolates were the prolines $(\mathrm{P})$ at amino acid positions 36, 37, 41, 44 (except BTV-8 (KJ506703)), 46, 50, 104, 157, 165, 220 and 227, confirming previous results suggesting conserved regions [36]. Isolates BTV-19 (KJ506699) and BTV-20 (KJ506702) had an additional proline (P) at amino acid 158, whereas BTV-8 (KJ506703) and BTV-S299 (KJ506704) have a serine (S) residue. The rest of the isolates had a glutamine (Q) at amino acids position 158. Two hydrophobic regions are conserved between amino acid positions 119 to 133 and 167 to 183 , respectively, with five viruses possessing a valine (V) instead of an isoleucine (I) residue at amino acid position 126 (Fig. 3). The amino acid region 110 to 130 had up to three amino acid changes in six of the strains, while region 149 to 200 had up to eight amino acid changes (e.g. BTV19 (KJ506699)) (Fig. 3). BTV-8 (KJ506703) and BTV-S299 (KJ506704) both had the most amino acids changes when compared to BTV-11 (KJ506694) i.e. 13 changes each (Fig. 3). These two viruses demonstrated the same amino acid changes, except at position 44 where BTV-8 (KJ506703) possess a leucine (L) and at position 45 where BTV-S299 (KJ506704) possesses a threonine (T). Amino acid position 154 differs in BTV-8 (KJ506703), BTV-S299 (KJ506704), BTV-23 (KJ506700), BTV-21 (KJ506701) and BTV-19 (KJ506699) replacing the alanine (A) residue with a threonine (T). Amino acid position 177 differs between the virus $\quad 1$ BTV-20 (KJ506702) and BTV-21 (KJ506701) the leucine (L) residue is replaced with an isoleucine (I) and in BTV-23 (KJ506700) it is replaced with a valine (V) (Fig. 3). Five viruses also demonstrated amino acid differences at position 187 where BTV-23 (KJ506700), BTV-21 (KJ506701) and BTV-20 (KJ506702) have a glycine (G) and BTV-8 (KJ506703), and BTV-S299 (KJ506704) an alanine (A) instead of a serine (S) residue (Fig. 3). 
BTV-11 (KJ506694) BTV-7 (KJ506695) BTV-2 (KJ506696) BTV-16 (KJ506697) BTV-22 (KJ506698) BTV-19 (KJ506699) BTV-23 (KJ506700) BTV-21 (KJ506701) BTV-20 (KJ506702) BTV-8 (KJ506703) BTV-S299 (KJ506704

BTV-11 (KJ506694) BTV-7 (KJ506695) BTV-2 (KJ506696) BTV-16 (KJ506697) BTV-22 (KJ506698) BTV-19(RJ506699) BTV -23 (KJ506700) BTV-21 (KJ506701) BTV-20 (KJ506702) BTV-8 (KJ506703) BTV-S299 (KJ506704)

BTV-11 (KJ506694) BTV-7 (KJ506695) BTV-2 (KJ506696) BTV-16 (KJ506697) BTV-22 (KJ506698) BTV-19 (KJ506699) BTV-23 (KJ506700) BTV-21 (KJ506701 BTV-20 (KJ506702) BTV-8 (KJ506703) BTV-S299 (KJ506704)

BTV-11 (KJ506694) BTV-7 (KJ506695) BTV-2 (KJ506696) BTV-16 (KJ506697) BTV-22 (KJ506698) BTV-1 9 (KJ506699) BTV -23 (KJ506700) BTV-21 (KJ506701) BTV-20 (KJ506702) BTV-8 (KJ506703) BTV-S299(KJ506704

BTV-11 (KJ506694) BTV-7 (KJ506695) BTV-2 (KJ506696)

BTV-16 (KJ506697) BTV-22 (KJ506698) BTV-19 (KJ506699) BTV-23 (KJ506700) BTV-21 (KJ506701) BTV-20 (KJ506702) BTV-8 (KJ506703) BTV-S299 (KJ506704)
10 20 30 40 50
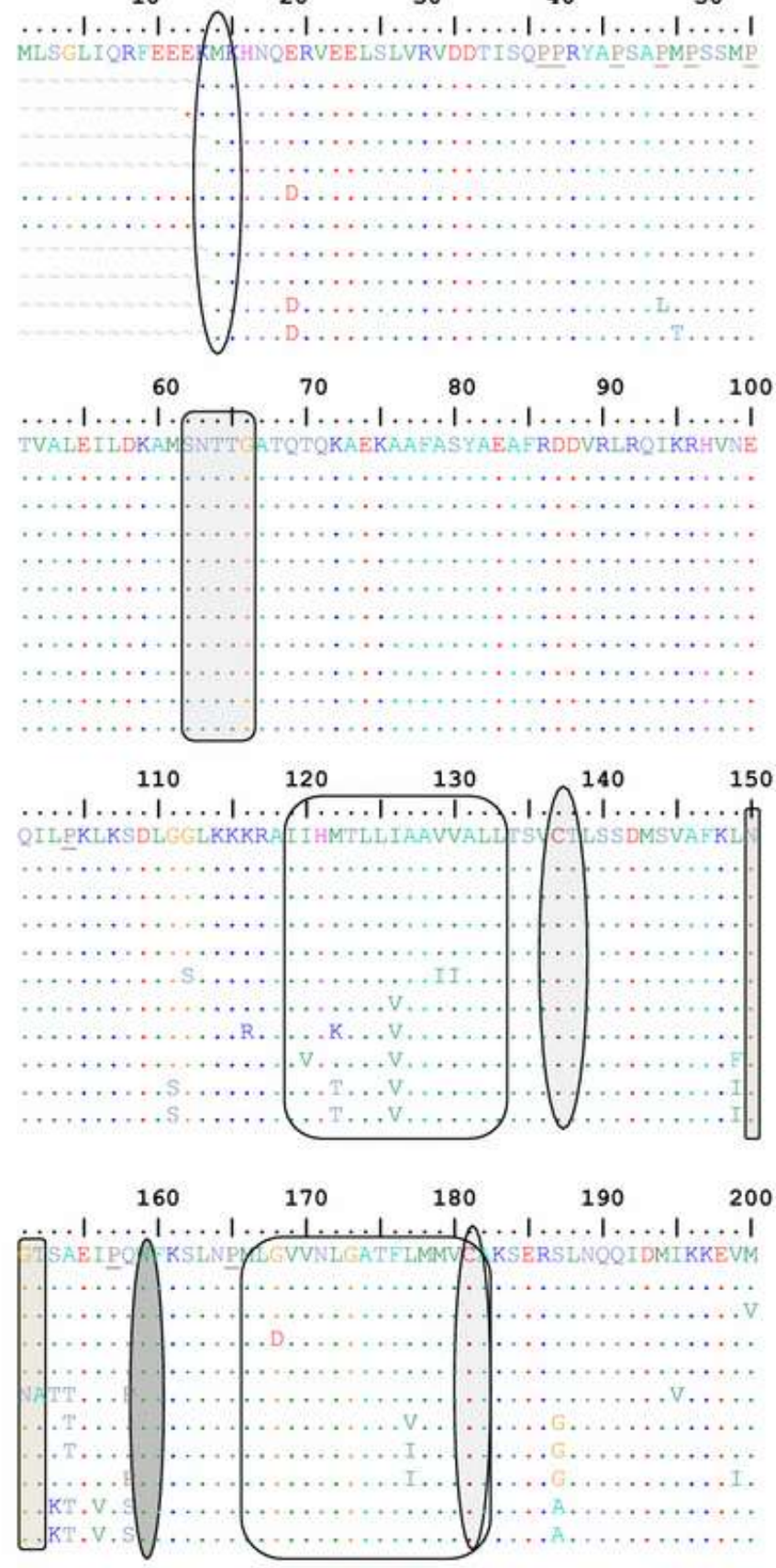

210 220

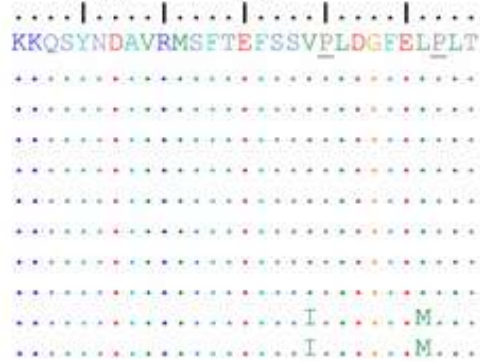

Fig. 3 : Amino acid sequence alignment, with conserved regions, of NS3/A from 11 BTV isolates. The NS3/A start codon (aa 14) is indicated by an oval. The proline residues (aa 36, 37, 41, 46, 50, 104, 157, 165, 220 and 227) are underlined. The two N-linked glycosylation sites (aa 62-66 and 150-152) are indicated by light grey shaded rectangles. Hydrophobic sites (aa 119-133 and 167-183) are indicated by open rectangles. The conserved cysteines (C) at amino acid positions 137 and 181 are indicated by light grey-shaded ovals, and the tryptophan (W) residue at amino acid position 157 is indicated by a dark greyshaded oval. Dots indicate residues that are identical in all 11 isolates 


\section{Discussion}

Previous studies have shown considerable sequence identity and conservation of the NS3/A protein encoded by segment 10 of the BTV viral genome, especially at amino acid level (36, 37, 31, 30, 20]. It was demonstrated that BTV strains can be divided into five groups/topotypes using the NS3/A protein/sequence data [30, 20]. These groups consisting of three major lineages, i.e. one Eastern group and two Western groups and two additional minor lineages of western and eastern origin. Phylogenetic network analyses (Fig. 1, Fig. 2) revealed that the three major groups contain the majority of the segment 10 sequences with fewer sequences in the two minor groups $[30,20,16]$. From both the NJ tree and the neighbour-net graph it was possible to determine the origin of the strains sequenced in this study. The BTV strains used in the study were isolated between 1937 and 2012. The isolation dates of the strains do not appear to influence the nucleotide identity between newly sequenced strains and global BTV strains (Table I).

Bluetongue virus 8 (KJ506703) and BTV-S299 (KJ506704) clustered into Western group 1 (Fig. 1c, Fig. 2c). Bluetongue virus S299 is closely related (96.86\%) to BTV-2 strains from SA (including a vaccine strain) and a BTV-2 strain from Portugal. This could be the result of reassortment between the SA BTV-2 MLV strain and a BTV-2 strain from Portugal. Bluetongue virus serotype 2 vaccine strains were isolated in both Italy and Portugal $[38,39]$.

Neighbour-net analysis does not support the split of BTV-8 (KJ506703) and BTV-7 (AY857506) into a separate cluster (Fig. 1) but rather clustered them in Western group 1 (Fig. 2c). This was also evident in the nucleotide differences between BTV-8 (KJ506703) and strains from Western group 2 (80.97\%) (Table I). The high nucleotide identity between BTV-8 (KJ506703) and BTV-7 (AY857506) (96.70\%) indicated the impact of genetic drift and/or reassortment between different serotypes [40 - 42]. The segment 10 gene sequences of BTV-2 (KJ506696), BTV-7 (KJ506695), BTV-11 (KJ506694), BTV-16 (KJ506697) and BTV-22 (KJ506698) all clustered into Western group 2 (Fig. 1a, Fig. 2a). These serotypes, along with BTV-8 (KJ506703) and BTV-7 (AY857506) from Western group 1, are all endemic to South Africa although they formed two separate groups/topotypes. This demonstrates that BTV topotypes are influenced by nucleotide similarities between the segment 10 genes of different BTV genomes.

The presence of BTV-16 (KJ506697) in Western group 2 is unexpected (Fig. 1a). This serotype is endemic to the Mediterranean Basin and Asia, with a few isolates from Australia. The latter strain has a nucleotide identity of $96.70 \%$ to BTV-7 from South Africa (KJ506695 and JX272558) and a BTV-11 reference strain (JQ972840) from Belgium (Table I). Gene reassortment between serotype 16 and/or BTV-7 and BTV-11 could explain the unexpected grouping. A double reassortant field isolate of BTV-16 was also isolated in 2002 in Italy, which contained a segment 2 derived from a SA BTV-16 MLV and a segment 5 derived from a SA BTV-2 MLV strain $(38,22,42]$. The neighbour-net analysis verifies the grouping of BTV-16 in the Western group 2 but rather illustrates reassortment between BTV-2 (KJ506696) which, in turn, is closely related to BTV-7 (KJ506695 and JX272558) (Fig. 2a).

Bootstrap values and split network analysis supports the clustering of the segment 10 genes from two South African BTV-19 strains (KJ506699 and JX2724389) into Western group 3 (Fig. 1a, Fig. 2d). Serotype 19 is 
endemic to both South Africa and the New World, and showed 100\% nucleotide identity (Table I). In both Western groups 1 and 2, segment 10 sequences obtained from isolates from the New World and Europe clustered together with South African strains. The clustering of the New World strains and South African strains was first described by van Niekerk et al., [31]. This appears to demonstrate the historical facilitated transfer of South African strains into the New World and suggests a history of genetic continuity between the strains [31, 30].

In Eastern group 1, strains isolated in the Mediterranean Basin (Greece) and Asia clustered together with Australian strains (Fig. 1b, Fig. 2b). The clustering of segment 10 from BTV-20 (KJ506702), BTV-21 (KJ506701) and BTV-23 (KJ506700) into an Eastern group is expected. These serotypes are South African exotics [43] and originated in Australia [44, 45], Asia [46, 47] or South America [48]. Bluetongue virus serotype 20 (KJ506702) demonstrated 100\% nucleotide identity to two Australian strains of BTV-20 (Table I). The segment 10 nucleotide sequence from BTV-21 (KJ506701) was 97.80\% identical to that of a serotype 2 strain from Australia, while BTV-23 (KJ506700) was 99\% identical to a serotype 2 strain from South Africa from Eastern group 1 (Table I, Fig. 1b, Fig. 2b). This suggests that BTV-23 circulated in South Africa in the past and that gene reassortment between the latter two strains occurred. Clustering of BTV-23 into Eastern group 1 could clarify the exotic status of this serotype in South Africa, although phylogenetic analyses of segment 2 are needed to be used to confirm this. No published data of BTV-23 isolated in South Africa in the last 20 years could be found. Conversely, previous studies demonstrated that geographical origin has little or no influence on the variation in the NS3/A gene [31].

The neighbour-net analysis provides strong evidence for networked evolution in segment 10 gene sequences of BTV isolates (Fig. 2). The analysis demonstrated reassortment of BTV segment 10 within the different topotypes. It highlighted the role that vaccine strains play in the epidemiology of BTV, e.g. BTV vaccine strain 2 from SA (JN255941), BTV-2 from India (JQ904064) and BTV-2 from Portugal (EF434179) all demonstrated similar splits probably due to reassortment of the three strains (Fig. 2c). Reassortment of genes could be due to co-circulation of strains and/or co-infection of either vector or host.

Five conserved regions of segment 10 identified previously were present in all 11 newly sequenced BTV isolates analysed $[36,37,31]$. These regions included the cysteine (C) at amino acid positions 137 and 181, and the tryptophan (W) residue at amino acid 159. Furthermore, two potential N-linked glycosylation sites at amino acid position 63 to 65 (NTT) and 150 to 152 (NGT) [36, 37] were present. The hydrophobic regions, N-linked glycosylation as well as the proline-rich regions were also conserved in all 11 isolates. The amino acid variation of NS3/A between strains complimented the phylogenetic networks inferred from the nucleotide sequences. Amino acid changes appear to be topotype-associated, with similar changes in closely related strains. Segments 10 encoding NS3/A from BTV-8 (KJ506703) and BTV-S299 (KJ506704) had the most amino acid differences of all 11 sequences. Differences in amino acid sequences could be a result of nucleotide substitution or nonsynonymous single nucleotide polymorphism caused by genetic reassortment or mutation $[37,21,30]$.

The implication of the differences between non-conserved amino acids with regards to biological properties such as virulence and the ability to be transmitted between the vector and the host needs to be examined. Adaptive selection is not evident [30] but corresponding amino acid differences within topotypes could be due 
to genetic drift of co-circulating viruses in a host-specific fashion [49]. The conserved nature of the segment 10 gene suggests that the encoded protein is critical to the biological function and the fitness of the virus in this ecosystem [37].

Phylogenetic analyses of segment 2 or segment 6 could be used to confirm the results as segment 2 (VP2) and segment 6 (VP5) also show variations within each serotype that correlate with the geographic origin of the virus strain $[50,51,6,52]$. These segments also show high levels of sequence variation that correlate with virus serotype [6, 52]. Genome segment 6 recently demonstrated a novel ninth topotype after identifying BTV serotype 26 (KUW2010/02) [5, 53]. This contradicts the five topotypes found when using segment 10 to geographically group isolates although the major and minor groups are similar, segment 10 lacks subgroups. Future studies should rather rely on full genome sequence of viruses as this could identify serotype, geographic origin (topotypes) and reassortment of genes.

\section{Conclusion}

Due to negative selection pressure such as reassortment, genetic drift and/or the founder effect on the BTV NS3/A encoding gene, it was suggested to classify BTV into topotypes [28, 37] This allowed for viral strains to be assigned to a specific geographical region based on conserved gene regions such as NS3. In this study, newly sequenced BTV field strains were compared to segment 10 sequences from global BTV isolates. Phylogenetic network analyses (neighbour-joining and neighbour-net) of 11 BTV segment 10 sequences revealed five topotypes [30, 20]. The newly sequenced isolates also demonstrated the five previously identified conserved regions $[31,20]$ with intermediate nucleotide identity (>79\%) [20]. Non-structural protein NS3/A shows very little overall variation within the proteins of global BTV strains.

\section{Acknowledgements}

The University of Pretoria, Department Veterinary Tropical Diseases for giving us the opportunity to do the work. Dr Melvyn Quan, Mr Louwrens Snyman, Dr Peter Coetzee and Miss Karen Eberhson for their assistance and Dr Otto Koekemoer for information on sequences. Funding form the National Research Foundation and the Meat Industry Trust.

\section{Competing interests}

The authors declare that they have no financial or personal relationships which may have inappropriately influenced the outcomes and conclusions they may have drawn.

\section{References}

1. Spreull, J. (1905). Malarial catarrhal fever (bluetongue) of sheep in South Africa. J Comp Pathol Therap, 18, 321337. 
2. Borden, E.C., Shoorijeh, S.J. \& Murphy, F.A. (1971). Physiochemical and morphological relationships of some athropod-borne viruses to bluetongue virus - a new taxonomic group. Physiochemical and serological studies. J Gen Virol, 13, 261-271.

3. Davies, F.G., Mungai, J.N \& Pini, A. (1992). A new bluetongue virus serotype isolated in Kenya. Vet Microbiol, $31,25-32$.

4. Hofmann, M.A., Renzullo, S., Mader, M., Chaignat, V., Worwa, G., et al. (2008). Genetic characterization of toggenburg orbivirus, a new bluetongue virus, from goats, Switzerland. Emerg Infect Dis, 14, 1855-1861.

5. Maan, S., Maan, N.S., Nomikou, K., Batten, C., Antony, F., et al. (2011). Novel bluetongue virus serotype from kuwait. Emerg Infect Dis, 17, 886-889.

6. Maan, S., Maan, N.S., Samuel, A.R., Rao, S.J., Attoui, H. \& Mertens, P.P.C. (2007). Analysis and phylogenetic comparisons of full-length VP2 genes of the 24 bluetongue virus serotypes. J Gen Virol, 88, 621-630.

7. Du Toit, R.M. (1944). The transmission of Bluetongue and horsesickness by Culicoides. Onderstepoort Jour Vet Sei and Anim Indus, 19, 7-16.

8. De Clercq, K., De Leeuw, I., Verheyden, B., Vandemeulebroucke, E., Vanbinst, T., et al. (2008). Transplacental infection and apparently immunotolerance induced by a wild-type bluetongue virus serotype 8 natural infection. Transbound Emerg Dis, 352-35.

9. Desmecht, D., Bergh, R.V., Sartelet, A., Leclerc, M., Mignot, C., et al. (2008). Evidence for transplacental transmission of the current wild-type strain of bluetongue virus serotype 8 in cattle. Vet Rec, 163, 50-52.

10. Vercauteren, G., Miry, C., Vandenbussche, F., Ducatelle,R., Van der Heyden, S., et al. (2008). Bluetongue virus serotype 8-associated congenital hydranencephaly in calves Transbound Emerg Dis, 55, 293-298.

11. Menzies, F.D., McCullough, S.J., McKeown, I.M., Forster, J.L., Jess, S. C., et al. (2008). Evidence for transplacental and contact transmission of bluetongue virus in cattle. Vet Rec, 163, 203-209.

12. Miller, P.J., Kim, L.M., I.P, H.S \& Afonson, C.L. (2009). Evolutionary dynamics of newcastle disease virus. Virology, 391, 64-72.

13. He, C., Xie, Z., Han, G., Dong, J., Wang, D., Liu, J., Ma, L., Tang, X., Liu, X., Pang, Y., et al. (2009). Homologous recombination as an evolutionary force in the avian influenza A virus. Mol Biol Evol, 26,177-187.

14. Knowles, N.J. \& Samuel, A.R. (2003). Molecular epidemiology of foot-and-mouth disease virus. Virus Res, 91, 65-80.

15. Holmes, E.C., Worobey, M. \& Rambaut, A. (1999). Phylogenetic evidence for recombination in dengue virus. Mol Biol Evol, 16, 405-409.

16. Maan, S., Maan, N.S., van Rijn, P.A, van Gennip, R.G.P., Sanders, A., et al. (2009). Full Genome characterisation of bluetongue virus serotype 6 from the Netherlands 2008 and comparison to other field and vaccine strains. PLOS ONE 5(4): e10323. doi:10.1371/journal.pone.0010323.

17. Mellor, P.S., Carpenter, S., Harrup, L., Baylis, M., Wilson, A. \& Mertens, P.P.C. (2009). Bluetongue in Europe and the Mediterranean Basin. In: Mellor, P.S,. Baylis, M,. Mertens, P.P.C (ed) Bluetongue, Elsevier. London, pp $235-264$.

18. Vandenbussche, F., De Leeuw, I., Vandemeulebroucke, E. \& De Clercq, K. (2009). Emergence of bluetongue serotypes in Europe, Part 1: description and validation of four real-time RT-PCR Assays for the serotyping of bluetongue viruses BTV-1, BTV-6, BTV-8 and BTV-11. Transbound Emerg Dis, 56, 346-354.

19. Huson, D.H. \& Bryant, D. (2006). Application of phylogenetic networks in evolutionary studies. In: Society for Molecular Biology and Evolution, Published by Oxford University Press, pp 254-267.

20. Maan, S., Maan, N.S., Ross-Smith, N., Batten, C.A., Shaw, A.E., Anthony, S.J., Samuel, A.R., Darpel, K.E., Veronesi, E., Oura, C.A.L., et al. (2008). Sequence analysis of bluetongue virus serotype 8 from the Netherlands 2006 and comparison to other European strains. Virology, 377, 308-318. 
21. Bonneau, K.R., Mullens, B.A. \& Maclachlan, N.J. (2001). Occurrence of genetic drift and founder effect during quasispecies evolution of the VP2 and NS3/NS3A genes of bluetongue virus upon passage between sheep, cattle, and Culicoides sonorensis. J Virol, 75, 8298-8305.

22. Batten, C.A., Maan, S., Shaw, A.E., Maan, N.S. \& Mertens, P.P.C. (2008). A European field strain of bluetongue virus derived from two parental vaccine strains by genome segment reassortment. Virus Res, 137, 56-63.

23. Samal, S.K., el-Hussein, A., Holbrook, F.R., Beaty, B.J. \& Ramig, R.F. (1987). Mixed infection of Culicoides variipennis with bluetongue virus serotypes 10 and 17: evidence for high frequency reassortment in the vector. $\mathrm{J}$ Gen Virol, 68, 2319-2329.

24. Scott, T. W., Weaver, S. C. \& Mallampalli, V. L. (1994). Evolution of mosquito-borne viruses. In: Morse, S. S (ed) Evolutionary Biology of Viruses, Raven Press, New York, pp 293-324,

25. Novella, I. S., Hershey, C. L., Escarmis, C., Domingo, E. \& Holland J. J. (1999). Lack of evolutionary stasis during alternating replication of an arbovirus in insect and mammalian cells. J Mol Biol, 287, 459-465.

26. Weaver, S. C., Brault, A. C., Kang, W. \& Holland J. J. (1999). Genetic and fitness changes accompanying adaptation of an arbovirus to vertebrate and invertebrate cells. J Virol, 73, 4316-4326.

27. Wirblich, C., Bhattacharya, B. \& Roy, P. (2006). Nonstructural Protein 3 of bluetongue virus assists virus release by recruiting ESCRT-I protein Tsg101. J Virol, 80, 460-473. doi:10.1128/JVI.80.1.460.

28. Celma, C.C.P. \& Roy, P. (2009). A viral non-structural protein regulates bluetongue virus trafficking and release. J Virol, 83(13), 6806-6816. doi:10.1128/JVI.00263-09.

29. Gibbs, E.P. \& Greiner, E.C. (1994). The epidemiology of bluetongue. Comp Immunol Microb, 17, 207-220.

30. Balasuriya, U.B.R., Nadler, S.A., Wilson, W.C., Pritchard, L.I., Smythe, A.B., Savini, G., Monaco, F., De Santis, P., Zhang, N., Tabachnick. W.J., et al. (2008). The NS3 proteins of global strains of bluetongue virus evolve into regional topotypes through negative (purifying) selection. Vet Microbiol 126, 91-100.

31. Van Niekerk, M., Freeman, M., Paweska, J.T., Howell, P.G., Guthrie, A.J., Potgieter, A.C., Van Staden, V. \& Huisman, H. (2003). Variation in the NS3 gene and protein in South Africa isolates of bluetongue and equine encephalosis viruses. J Gen Virol, 84, 581-590.

32. Huismans, H., van Staden, V., Fick, W.C., van Niekerk, M. \& Meiring, T.L. (2004). A comparison of different orbivirus proteins that could affect virulence and pathogenesis. Veter Ital Ser, 40, 417-425.

33. Carpi, G., Holmes, E.C. \& Kitchen, A. (2010). The evolutionary dynamics of bluetongue virus. J Mol Evol, 70(6), 583-592.

34. Tamura, K. (1992). Estimation of the number of nucleotide substitutions when there are strong transitiontransversion and G+C content biases. Mol Biol Evol 9, 4, 678-687.

35. Bryant, D. \& Moulton, V. (2004). Neighbor-Net: an agglomerative algorithm for the construction of planar phylogenetic networks. Mol Biol Evol, 2, 255-265.

36. Hwang, G.Y., Yang, Y.Y., Chiou, J.F. \& Li, J.K. (1992). Sequence conservation among the cognate non-structural NS3:3A protein genes of six bluetongue viruses. Virus Res 23, 151-161.

37. Pierce C.M., Balasuriya, U.B. \& MacLachlan, N.J. (1998). Phylogenetic comparison of the S10 genes of field and laboratory strains of bluetongue virus from the United States. Virus Res, 55, 15-27.

38. Ferrari, G., De Liberato, C., Scavia, G., Lorenzetti, R., Zini, M., Farina, F., Magliano, A., Cardeti, G., Scholl, F., Guidoni, M., et al. (2005). Active circulation of bluetongue vaccine virus serotype-2 among unvaccinated cattle in central Italy. Prev Vet Med, 68 (2-4), 103-13.

39. Barros, S.C., Ramos, F., Luis, T.M., Vaz, A., Duarte, M., Henriques, M., Cruz, B. \& Fevereiro, M. (2007). Molecular epidemiology of bluetongue virus in Portugal during 2004-2006 outbreak. Vet Microbiol, 124, 25-34.

40. Huismans, H. \& Erasmus, B. J. (1981). Identification of the serotype-specific and group specific antigens of bluetongue virus. Onderstepoort J Vet, 48, 51-58. 
41. Mertens, P. P. C. (1999). Orbiviruses and coltiviruses - general features. In: Webster R.G and A. Granoff, A (Eds) Encyclopaedia of Virology, $2^{\text {nd }}$, London: Academic Press, pp. 1043-1061.

42. Listes, E., Monaco, F., Labrovic, A., Paladini, C., Leone, A., Di, G.L., Camma, C. \& Savini, G. (2009). First evidence of bluetongue virus serotype 16 in Croatia. Vet Microbiol, 138, 92-97.

43. Gerdes, G.H. (2004). A South African overview of the virus, vectors, surveillance and unique features of bluetongue. Veter Ital Ser, 40, 3, 39-42.

44. Delle-Porta, A.J., Sellers, R.F., Herniman, K.A., Littlejohns, I.R., Cybinski, D.H., St George, T.D., McPhee, D.A., Snowdon, W.A., Campbell, J., Cargill, C., et al. (1983). Serological studies of Australian and Papua New Guinean cattle and Australian sheep for the presence of antibodies against bluetongue group viruses. Vet Microbiol, 8, 147162.

45. St George, T.D., Cybinski, D.H., Della-Porta, A.J., McPhee, D.A., Wark, M.C. \& Bainbridge, M.H. (1980). The isolation of two bluetongue viruses from healthy cattle in Australia. Aust Vet J, 56, 562-563.

46. Sendow, I., Pritchard, L.I., Eaton, B.T. \& Daniels, P.W. (1997). Genotypic relationships of bluetongue viruses from Indonesia. Arbovirus Res Aust, 7, 263- 266.

47. Pritchard, L.I., Sendow, I., Lunt, R., Hassan, R., Kattenbelt, J., Gould, A.R., Daniels, P.W. \& Eaton, B.T. (2004). Genetic diversity of bluetongue viruses in south east Asia. Virus Res, 101, 193-201.

48. Cunha, R.G. (1990). Neutralizing antibodies for different serotypes of bluetongue virus in sera of domestic ruminants from Brazil. Rev Bras Med Vet, 12, 3-7.

49. Bonneau, K.R. \& MacLachlan, N.J. (2004). Genetic diversification of field strains of bluetongue virus. Veter Ital Ser, 40, 446-447.

50. Bonneau, KR., Zhang, N., Zhu, J., Zhang, F., Li, Z., et al. (1999). Sequence comparison of the L2 and S10 genes of bluetongue viruses from the United States and the People's Republic of China. Virus Res, 61,153-160.

51. Gould, A.R. \& Pritchard, L.I., (1990). Relationships amongst bluetongue viruses revealed by comparison of capsid and outer coat protein nucleotide sequences. Virus Res, 17, 31-52.

52. Singh, KP., Maan, S., Samuel, A.R., Rao, S., Meyer, A \& Mertens, P.P.C. (2004). Phylogenetic analysis of bluetongue virus genome segment 6 (encoding VP5) from different serotypes. Vet. Ital, 40, 479-483.

53. Maan, S., Maan, N.S., Nomikou, K., Veronesi, E., Bachanek-Bankowska, K., et al. (2011). Complete Genome Characterisation of a Novel 26th Bluetongue Virus Serotype from Kuwait. Plos ONE, DOI: 10.1371/journal.pone.0026147. 


\section{Appendix I}

The accession number, origin, year of isolation and reference of sequence data of the segment 10 gene of different BTV isolates obtained from GenBank and used to compare newly sequenced isolates from SA.

\begin{tabular}{|c|c|c|c|}
\hline $\begin{array}{l}\text { Accesion } \\
\text { number }\end{array}$ & Origin & $\begin{array}{c}\text { Year of } \\
\text { isolation/ } \\
\text { submitted }\end{array}$ & Reference \\
\hline AB473808 & Japan & 2008 & Inumaru and Hosamani, 2008 \\
\hline AF044373 & TX, USA & 1962 & Pierce et al. , 1998 \\
\hline $\mathrm{AFO} 44377$ & USA & 1998 & Pierce et al., 1998 \\
\hline $\mathrm{AFO} 44383$ & CAV Kern & 1981 & Pierce et al., 1998 \\
\hline AF044386 & NE/ Pershing & 1979 & Pierce et al., 1998 \\
\hline $\mathrm{AFO44702}$ & CA Kern & 1980 & Pierce et al., 1998 \\
\hline AF044703 & CAVKern & 1981 & Pierce et al., 1998 \\
\hline $\mathrm{AFO} 44704$ & CAKern & 1981 & Pierce et al., 1998 \\
\hline AF481093 & Corsican, France & 2001 & Sailleau et al., unpublished \\
\hline AF481094 & SA & 2002 & Breard et al., 2001 \\
\hline AF512913 & India & 1999 & van Niekerk et al., 2003 \\
\hline AF512916 & Ermelo, Mpumalanga, SA & 1958 & van Niekerk et al., 2003 \\
\hline AF512919 & SA & 2002 & van Niekerk et al., 2003 \\
\hline AF512920. & Free State, SA & 1999 & van Niekerk et al., 2003 \\
\hline AF512921 & Gauteng, SA & 1999 & van Niekerk et al., 2003 \\
\hline AF512922 & Beaufort West, Western Cape, SA & 1944 & van Niekerk et al., 2003 \\
\hline AF512923 & Nelspoort, Western Cape, SA & 1944 & van Niekerk et al., 2003 \\
\hline AF512924 & Onderstepoort, SA & 1937 & van Niekerk et al., 2003 \\
\hline AY120938 & SA & 2002 & van Niekerk et al., 2003 \\
\hline AY426604 & Caribbean & 1990 & Balasuriya et al., unpublished \\
\hline AY449652 & Greece & 2003 & Nikolakaki et al., 2005 \\
\hline AY775164 & SA & 2004 & Monaco et al., 2006 \\
\hline AY857506 & SA & 2004 & Breard et al., 2007 \\
\hline EF434179 & Portugal & 2005 & Barros et al.,2007 \\
\hline EU131022 & India & 2007 & Desai et al., 2009 \\
\hline EU131027 & India & 2007 & Desai et al., 2009 \\
\hline FJ183383 & Netherlands & 2008 & Potgieter et al., 2009 \\
\hline FJ713322 & USA & 2009 & Wilson et al., 2009 \\
\hline GQ506460 & Netherlands & 2007 & Maan et al., 2012 \\
\hline JN255871 & Italy & 2000 & Caporale et al., 2011 \\
\hline JN255931 & SA (reference strain/wild type) & 2001 & Caporale et al., 2011 \\
\hline JN255941 & SA (Vacc) & 2000 & Caporale et al., 2011 \\
\hline JN671915 & China & 1996 & Yang et al., 2011 \\
\hline JQ086240 & Beatrice Hill/CPRS, NT, Australia & 1986 & Boyle et al., 2012 \\
\hline JQ086250 & Douglas Daly, NT, Australia & 2008 & Boyle et al., 2012 \\
\hline JQ086260 & Beatrice Hill/CPRS, NT, Australia & 1977 & Boyle et al., 2012 \\
\hline JQ086270 & VRRS, NT, Australia & 1979 & Boyle et al., 2012 \\
\hline JQ086280 & TFRF, NT & 1982 & Boyle et al., 2012 \\
\hline JQ086300 & Beatrice Hill/CPRS, NT, Australia & 2007 & Boyle et al., 2012 \\
\hline JQ240330 & Australia & 2010 & Boyle et al., 2012 \\
\hline JQ713563 & India & 1982 & Maan et al., 2012 \\
\hline JQ904064 & India & 2008 & Minakshi et al., unpublished \\
\hline JQ972840 & Belgium & 2010 & Vandenbussche et al., in press \\
\hline JQ972850 & Belgium & 2010 & Vandenbussche et al., in press \\
\hline JQ972860 & Germany & 2011 & Vandenbussche et al., in press \\
\hline JQ972870 & Caribbean & 2010 & Vandenbussche et al., in press \\
\hline JX129386 & Pakistan & 2012 & Maan et al., 2012 \\
\hline JX272398 & SA (Culicoides pools) & 2012 & Koekemoer et al., unpublished \\
\hline$J \times 272418$ & Australia & 1979 & Koekemoer et al., unpublished \\
\hline$J \times 272428$ & Australia & 1975 & Koekemoer et al., unpublished \\
\hline$J \times 272438$ & SA & 1976 & Koekemoer et al., unpublished \\
\hline JX272468 & Hazara, West Pakistan & 1960 & Koekemoer et al., unpublished \\
\hline$J \times 272518$ & Nelspoort, Cape Province, SA & 1944 & Koekemoer et al., unpublished \\
\hline JX272548 & Onderstepoort, Transvaal, SA & 1937 & Koekemoer et al., unpublished \\
\hline$J \times 272558$ & Utrecht, Natal, SA & 1955 & Koekemoer et al., unpublished \\
\hline JX272608 & Ermelo, Transvaal, SA & 1959 & Koekemoer et al., unpublished \\
\hline
\end{tabular}




\section{Appendix II}

Bluetongue virus isolates obtained from 1972 to 2000 in South Africa, the isolation date and GenBank accession number, indicating sequence data of segment 10 for all isolates.

\begin{tabular}{ccc}
\hline Strain & $\begin{array}{c}\text { Year received at } \\
\text { DVTD, OP }\end{array}$ & Accession number \\
\hline BTV-2_NS3/A & 1972 & KJ506696 \\
BTV-7_NS3/A & 1972 & KJ506695 \\
BTV-8_NS3/A & 1972 & KJ506703 \\
BTV-11_NS3/A & 1972 & KJ506694 \\
BTV-16_NS3/A & 1972 & KJ506697 \\
BTV-19_NS3/A & 1995 & KJ506699 \\
BTV-20_NS3/A & 1995 & KJ506702 \\
BTV-21_NS3/A & 1995 & KJ506701 \\
BTV-22_NS3/A & 1995 & KJ506698 \\
BTV-23_NS3/A & 1995 & KJ506700 \\
BTV-S299_NS3/A & 2000 & KJ506704
\end{tabular}

